\title{
Sobre a unicidade da solução no controle de Sistemas Lineares com Saltos Markovianos não observados
}

\author{
Larissa T. Oliveira* Eduardo F. Costa \\ Depto. de Matemática Aplicada e Estatística, ICMC, USP, \\ Av. Trabalhador são-carlense, 400, Centro, CEP: 13566-590, São Carlos, SP \\ E-mail: 1tdo@icmc.usp.br, efcosta@icmc.usp.br.
}

\section{$\underline{\text { RESUMO }}$}

Os Sistemas Lineares com Saltos Markovianos (SLSM) são considerados uma importante classe de sistemas e vêm sendo intensamente estudados, principalmente pela característica de serem similares a sistemas lineares determinísticos clássicos e por modelar sistemas que possuem alterações abruptas em sua dinâmica em determinados instantes. Os SLSMs têm aplicações em várias áreas como economia [1, 2] e engenharia (por exemplo em robótica [4, 9] e na fabricação de papel [3]).

Um dos métodos disponíveis na literatura para resolver o problema de custo quadrático de $N$ estágios para sistemas lineares com saltos é comumente chamado de método variacional [5]. Este método baseiase em uma condição do tipo "gradiente nulo", que é necessária porém não suficiente para otimalidade da solução, conforme apresentado em [7]. Surgiram, em seguida, alguns trabalhos que conjecturaram sobre a suficiência desta condição, como por exemplo [8], sem chegar contudo a uma conclusão definitiva; mais recentemente foi observado que a questão continua em aberto [6].

Uma formulação sucinta do problema é como segue. Seja uma cadeia de Markov cujo estado no "instante" $k \geq 0$ é denotado por $\theta(k)$ e que toma valores em $\mathcal{T}=\{1,2, \cdots, T\}$. Assuma $P=\left[p_{i j}\right]$ conhecida, tal que $p_{i j}=\operatorname{Pr}\{\theta(k+1)=j \mid \theta(k)=i\}$; além disso, seja $P(\theta(k)=i)=\pi_{i}(k)$. Associado a esta cadeia, considere a seguinte recursão: $X_{i}(k)=\sum_{j=1}^{T} p_{j i}\left(A_{j}+B_{j} g(k)\right) X_{j}(k-1)\left(A_{j}+B_{j} g(k)\right)^{\prime}+$ $\sum_{j=1}^{T} p_{j i} \pi_{j}(k-1) G_{j} G_{j}^{\prime}$, com condição inicial dada por $X_{i}(0)=\pi_{i}(0) x(0) x^{\prime}(0)$, na qual as matrizes $A_{j}, B_{j}$ e $G_{j}, j \in \mathcal{T}$, são conhecidas e de dimensões apropriadas, e $g(k)$ é a variável (matricial) a ser determinada. Considerando matrizes de ponderação positivas semidefinidas $C_{j}, D_{j}, j \in \mathcal{T}$, definimos o problema de otimização na variável $g=\{g(0), g(1), \ldots, g(N-1)\}$ :

$$
\min _{g} \mathcal{J}_{N}=\sum_{k=0}^{N-1} \sum_{i=1}^{T} \operatorname{tr}\left\{\left(C_{i}+g^{\prime}(k) D_{i} g(k)\right) X_{i}(k)\right\} .
$$

Para a interpretação física e detalhes do problema acima, por gentileza consulte [2, 6, 7]. O valor de $\theta(k)$ não é observado, explicando porque o problema acima não depende dessa variável.

A contribuição deste resumo é apresentar um exemplo numérico esclarecendo que o problema pode apresentar mínimos locais múltiplos e distintos, levando ao resultado formalizado no final deste resumo. Em particular, conclui-se que a condição de otimalidade citada no início não é suficiente para otimalidade; também, o método variacional pode convergir para diferentes mínimos locais, como ilustrado no exemplo. Este exemplo é um dos dois que encontramos entre 10.000 gerados aleatoriamente.

Exemplo numérico. Considere os parâmetros:

$$
\begin{aligned}
& A_{1}=\left[\begin{array}{cc}
0.3182 & -0.4145 \\
0.5992 & 1.3150
\end{array}\right], \quad A_{2}=\left[\begin{array}{cc}
-1.6236 & -0.1252 \\
1.6573 & -2.5347
\end{array}\right], \quad A_{3}=\left[\begin{array}{cc}
-1.5097 & 2.7404 \\
-3.8474 & 2.7029
\end{array}\right], \quad A_{4}=\left[\begin{array}{cc}
-24.5608 & 35.9306 \\
-12.6778 & 18.0155
\end{array}\right] \text {, } \\
& C_{1}=\left[\begin{array}{cc}
2.2161 & -2.5044 \\
-2.5044 & 2.8302
\end{array}\right], C_{2}=\left[\begin{array}{ll}
0.0037 & 0.0808 \\
0.0808 & 1.7709
\end{array}\right], C_{3}=\left[\begin{array}{cc}
0.4955 & -0.4143 \\
-0.4143 & 0.3464
\end{array}\right], C_{4}=\left[\begin{array}{cc}
9.2357 & -12.4190 \\
-12.4190 & 16.6996
\end{array}\right], \\
& B_{1}=\left[\begin{array}{l}
-0.7975 \\
-0.1901
\end{array}\right], \quad B_{2}=\left[\begin{array}{c}
-0.1653 \\
0.7806
\end{array}\right], \quad B_{3}=\left[\begin{array}{c}
0.3732 \\
-0.2935
\end{array}\right], \quad B_{4}=\left[\begin{array}{l}
-0.7853 \\
-0.4005
\end{array}\right], \quad G_{1}=\left[\begin{array}{c}
2.4078 \\
0.7081
\end{array}\right], \quad G_{2}=\left[\begin{array}{c}
0.7858 \\
-0.8289
\end{array}\right] \text {, } \\
& G_{3}=\left[\begin{array}{l}
0.5203 \\
0.3073
\end{array}\right], G_{4}=\left[\begin{array}{l}
-0.5039 \\
-0.2670
\end{array}\right], \pi(0)=\left[\begin{array}{llll}
0.2440 & 0.1691 & 0.5412 & 0.0457
\end{array}\right], x(0)=\left[\begin{array}{c}
-0.5212 \\
0.0605
\end{array}\right], \quad D_{1}=0.3613,
\end{aligned}
$$

\footnotetext{
*Agradecimentos a FAPESP procs.: 2010/12360-3, 2012/14085-5 e 2013/19380-8, CAPES procs.: DS-7899600/M e CNPq procs.: 311290/2013-2 e 306466/2010-4 pelos apoios financeiros concedidos.
} 
$D_{2}=0.4700, D_{3}=0.0588, D_{4}=0.3300, T=4, N=27$ e $P=\left[p_{i j}\right]$, onde $p_{11}=p_{22}=p_{33}=1, p_{42}=p_{43}=$ $0.2840, p_{44}=0.4321$ e os demais nulos.

Para obter os resultados apresentados a seguir implementamos o método variacional de [5] utilizando o software $M A T L A B^{\circledR}$ na versão 7.8. O método foi inicializado com duas sequências de ganhos iniciais distintas $g_{1}^{0}(k)=\left[\begin{array}{ll}0 & 0\end{array}\right]$ e $g_{2}^{0}(k)=\left[\begin{array}{ll}-2.1563 & 1.8474\end{array}\right]$, para $k=\{0, . ., N-1\}$, e tolerância $\epsilon=10^{-16}$.

A partir da sequência de ganhos $g_{1}^{0}$ obtivemos o valor ótimo de $\mathcal{J}_{N_{1}}^{*} \approx 2.72989 \times 10^{22}$ com ganho ótimo $g_{1}^{*}(N)=\left[\begin{array}{ll}-5.0153 & 1.3996\end{array}\right]$. Já com a sequência de ganhos $g_{2}^{0}$ obtivemos um valor ótimo inferior $\mathcal{J}_{N_{2}}^{*} \approx$ $1.36997 \times 10^{21}$ com ganho ótimo $g_{2}^{*}(N)=\left[\begin{array}{ll}-1.0400 & -0.6382\end{array}\right]$.

Nas figuras a seguir podemos ver as curvas de nível da função $\mathcal{J}_{N}$ nos pontos próximos aos ganhos ótimos locais $g_{1}^{*}(N)$ e $g_{2}^{*}(N)$, respectivamente. No eixo x estão os valores da primeira coordenada do vetor ganho e no eixo y os valores da segunda coordenada. Em ambas figuras destacamos o ponto ótimo.

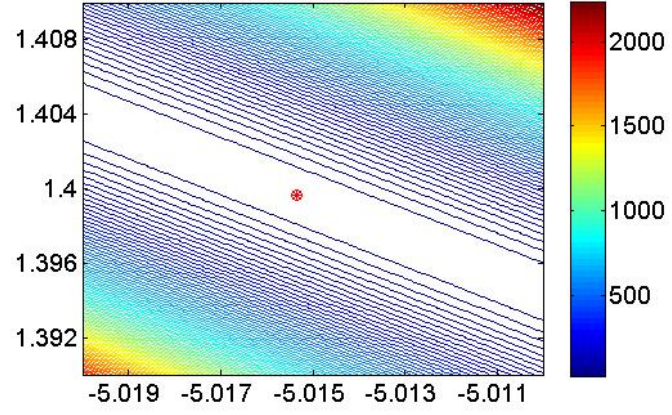

(a) Curvas de nível próximas ao ganho ótimo $g_{1}^{*}(N)$.

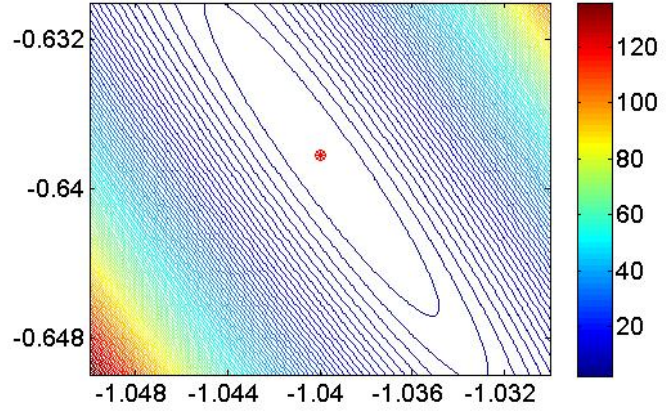

(b) Curvas de nível próximas ao ganho ótimo $g_{2}^{*}(N)$.

Concluímos este resumo formalizando o seguinte resultado.

Teorema 1. A solução ótima do problema de otimização (1) pode não ser única.

Palavras-chave: Sistemas Lineares com Saltos Markovianos, método variacional, unicidade de solução.

\section{Referências}

[1] O. L.V. COSTA and W. L. DE PAULO. Indefinite quadratic with linear costs optimal control of Markov jump with multiplicative noise systems. Automatica, 43(4):587 - 597, 2007.

[2] J. B. R. DO VAL and T. BASAR. Receding horizon control of jump linear systems and a macroeconomic policy problem. Journal of Economic Dynamics \& Control, 23:1099 - 1131, 1999.

[3] M. KHANBAGHI, R. P. MALHAME, and M. PERRIER. Optimal white water and broke recirculation policies in paper mills via jump linear quadratic control. IEEE Transactions on Control Systems Technology, 10(4):578 $-588,2002$.

[4] G. N. SARIDIS. Intelligent robotic control. IEEE Transactions on Automatic Control, 28:547 - 557, 1983.

[5] A. N. VARGAS. Controle por horizonte retrocedente de sistemas lineares com saltos Markovianos e ruído aditivo. Dissertação de mestrado, Faculdade de Engenharia Elétrica e de Computação, Universidade Estadual de Campinas, SP, 2004.

[6] A. N. VARGAS, E. F. COSTA, and J. B. R. DO VAL. On the control of Markov jump linear systems with no mode observation: application to a DC motor device. International Journal of Robust and Nonlinear Control, 23(10):1136 - 1150, 2013.

[7] A. N. VARGAS, J. B. R. DO VAL, and E. F. COSTA. Receding horizon control of Markov jump linear systems subject to noise and unobserved state chain. In 43rd IEEE Conference on Decision and Control, CDC, pages 4381 - 4386, 2004.

[8] A. N. VARGAS, J. B. R. DO VAL, and E. F. COSTA. Optimality condition for the receding horizon control of Markov jump linear systems with non-observed chain and linear feedback controls. In 44th IEEE Conference on Decision and Control and 2005 European Control Conference. CDC - ECC '05, pages 7308 - 7313, 2005.

[9] A. N. VARGAS, W. FURLONI, and J. B. R. DO VAL. Second moment constraints and the control problem of Markov jump linear systems. Numerical Linear Algebra with Applications, 20(2):357 - 368, 2013. 